\title{
The atomic method for the Hubbard dimer
}

\author{
Lira, R. N.*, Figueira, M. S. \\ Universidade Federal Fluminense \\ *rlira@id.uff.br
}

Keywords: Hubbard model, cumulant expansion, Green's functions

\section{Abstract}

The single-band Hubbard Hamiltonian [1] is the simplest model of interacting electrons in a lattice. It was developed by John Hubbard, who introduced the model to take into account the local electronic correlations in narrow energy bands. The model has a kinetic term that allows hopping of electrons between atomic sites and a term that considers the on-site interaction between electrons. This model is largely used in solid state physics to study magnetic properties of solids, insulator-metal transition (Mott transition) and high temperature superconductors. However, this is a problem that has exact analytical solution in a very few limiting cases and must be treated approximately or numerically.

In this work, we propose a new methodology to analytically solve the Hubbard Hamiltonian, mapping it into a two-site model (the Hubbard dimer). To obtain the Green's function for the lattice, we employed the cumulant expansion technique, using as a 'seed' the exact two-site Green's function (the atomic method) [2]. From the Green's function, we obtained the density of states [DOS] and the occupation numbers as functions of the external parameters of the model, and compared the results with the Hubbard I approximation [1].

\section{Introduction}

The single band Hubbard model is the simplest interacting particles model in a lattice: it is an extension of the tight-binding model, accounting for the electron-electron interaction between electrons on the same site.
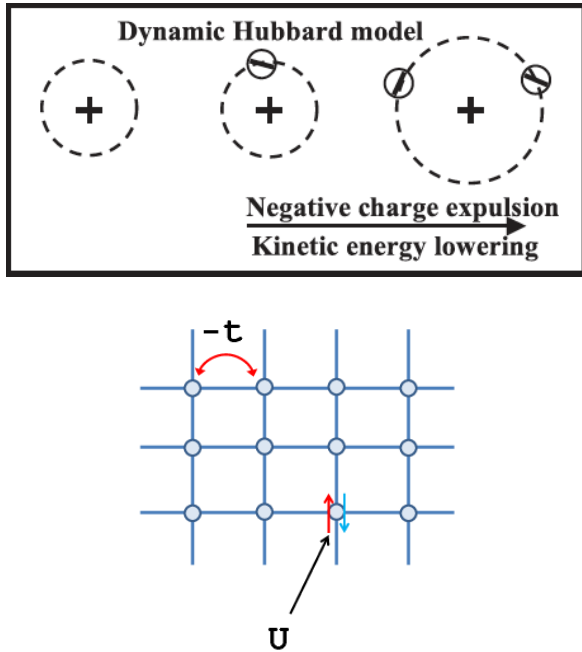

Figure 1. The interactions in the Hubbard model

18th Brazilian Workshop on Semiconductor Physics (BWSP 2017)
The microscopic model consists of two parts: a kinetic term describing the hopping of electrons and a second term which approximates the Coulomb interaction among them. From the original Hubbard work [1] we know that the Hamiltonian to model a lattice including the electron correlations is given by

$$
H=\epsilon_{0} \sum_{i \sigma} c_{i \sigma}^{\dagger} c_{i \sigma}+\sum_{i \neq j} t_{i j} c_{i \sigma}^{\dagger} c_{j \sigma}+\frac{U}{2} \sum_{i \sigma} n_{i \sigma} n_{i \bar{\sigma}}
$$

In this work we study the Hubbard dimer, which is modeled by the Hubbard Hamiltonian with only two sites. Each site might be empty (0), occupied by a spin up electron $(\uparrow)$ or a spin down electron $(\downarrow)$, or by two electrons with opposite spins $(\uparrow \downarrow)$. These states generate a Fock space for the dimer

This problem treats a non-trivial interacting electronic system that has exact analytical solution. The Hamiltonian used to model this problem is [2]:

$$
\begin{aligned}
H= & \epsilon_{0} \sum_{i=1,2} \sum_{\sigma} c_{i \sigma}^{\dagger} c_{i \sigma}-t \sum_{\substack{i \neq j \\
i, j=1,2}} \sum_{\sigma} c_{i \sigma}^{\dagger} c_{j \sigma}+ \\
& +\frac{u}{2} \sum_{i=1,2} \sum_{\sigma \sigma^{\prime}} c_{i \sigma}^{\dagger} c_{i \sigma^{\prime}}^{\dagger} c_{i \sigma^{\prime}} c_{i \sigma}
\end{aligned}
$$

where $t$ is the kinetic hopping energy of the electrons, which represents the possibility of an electron hopping from a site to another; $u$ denotes the 
Coulomb interaction between two electrons on the same site; $\varepsilon_{0}$ is the local energy of an electron.

\section{Methods and Results}

Calculating the sums and rewriting the Hamiltonian in the basis of the dimer states one gets a $16 \times 16$ matrix and by calculating its eigenvectors and eigenvalues, the complete solution of the model for the dimer can be found (table 1). From this solution, it is possible to identify the transitions on the dimer and the energy variation associated with these (table 2).

Table 1. The exact solution of the model for the dimer

\begin{tabular}{|c|c|c|c|c|}
\hline State & Eigenvectors $|n, r\rangle$ & Eigenvalues $E_{i}$ & $\mathrm{n}$ & $s_{z}$ \\
\hline $1=|0,0\rangle$ & $|0,1\rangle=|0,0\rangle$ & $E_{1}=0$ & 0 & 0 \\
\hline $2=|t, 0\rangle$ & $|1,1\rangle=\frac{1}{\sqrt{2}}(|t, 0\rangle-\mid 0, t)$ & $E_{2}=\epsilon_{0}+t$ & 1 & $+\frac{1}{2}$ \\
\hline $3=|\omega, 0\rangle$ & $|1,2\rangle=\frac{1}{\sqrt{2}}(|\downarrow, 0\rangle-\mid 0,4)$ & $E_{3}=\epsilon_{0}+t$ & 1 & $-\frac{1}{2}$ \\
\hline $4=(0, \pi)$ & $|1,3\rangle=\frac{1}{\sqrt{2}}(|t, 0\rangle+|0,7\rangle)$ & $E_{4}=\epsilon_{0}-t$ & 1 & $+\frac{1}{2}$ \\
\hline $5=(0,4)$ & $|1,4\rangle=\frac{1}{\sqrt{2}}(|1,0\rangle+(0,4)$ & $E_{5}=\epsilon_{0}-t$ & 1 & $-\frac{1}{2}$ \\
\hline $6=|t, \pi|$ & $R, 1\rangle=|t, \pi\rangle$ & $E_{6}=2 \epsilon_{0}$ & 2 & +1 \\
\hline $7=\mid \downarrow \downarrow \downarrow)$ & $(2,2\rangle=|\downarrow, \downarrow|)$ & $E_{7}=2 \epsilon_{0}$ & 2 & -1 \\
\hline $8=\mid t, 4)$ & $\left.(2,3\rangle=\frac{1}{\sqrt{2}}(\mid t, 4)+|\downarrow, t\rangle\right)$ & $E_{8}=2 \epsilon_{0}$ & 2 & 0 \\
\hline $9=|\omega t\rangle$ & $(2,4)=\frac{1}{\sqrt{2}}((0, \tau \downarrow)-(\uparrow \downarrow, 0))$ & $E_{9}=2 \epsilon_{0}+u$ & 2 & 0 \\
\hline $10=|\uparrow \downarrow 0\rangle$ & 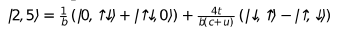 & $E_{10}=2 \epsilon_{0}+\frac{u+c}{2}$ & 2 & 0 \\
\hline $11=(0, T \psi)$ & $\left.(2,6)=\frac{1}{a}((0, T \downarrow)+(T \downarrow, 0\rangle)+\frac{4 t}{a(c-u)}(\mid \tau, \downarrow)-(\downarrow, T\rangle\right)$ & $E_{11}=2 \epsilon_{0}+\frac{u-c}{2}$ & 2 & 0 \\
\hline $12=|\tau b, \tau\rangle$ & $\left.\beta, 1\rangle=\frac{1}{\sqrt{2}}(|\tau, T \psi+| \tau \downarrow, \tau)\right)$ & $E_{12}=3 \epsilon_{0}+u-t$ & 3 & $+\frac{1}{2}$ \\
\hline $13=|\uparrow \downarrow, \downarrow\rangle \mid$ & $|3,2\rangle=\frac{1}{\sqrt{2}}(|\downarrow, \tau \downarrow\rangle+\mid \uparrow \downarrow, \downarrow \downarrow)$ & $E_{13}=3 \epsilon_{0}+u-t$ & 3 & $-\frac{1}{2}$ \\
\hline $14=|t, T t\rangle$ & $\langle, 3\rangle=\frac{1}{\sqrt{2}}(|\tau, \uparrow \downarrow\rangle-|\uparrow \downarrow, t\rangle)$ & $E_{14}=36_{0}+u+t$ & 3 & $+\frac{1}{2}$ \\
\hline $15=|\omega \uparrow \downarrow|$ & $\left.\langle 3,4\rangle=\frac{1}{\sqrt{2}}(|\downarrow, \uparrow \downarrow\rangle-\mid \uparrow \downarrow \downarrow \downarrow)\right)$ & $E_{15}=36_{0}+u+t$ & 3 & $-\frac{1}{2}$ \\
\hline $16=|\uparrow \downarrow \uparrow \downarrow\rangle \mid$ & $|4,1\rangle=|\uparrow \downarrow, \uparrow \downarrow\rangle$ & $E_{16}=4 \epsilon_{0}+u$ & 4 & 0 \\
\hline
\end{tabular}

Thesixteen eigenvectors $\langle n, r\rangle$ have energies $E_{i}$; being $n$ the number of electrons, $r$ the state of the dimer with $n$ electrons and $S_{z}$ the total spin in the $z$ direction. Also, $a=\sqrt{2\left(\left(\frac{16 t^{2}}{(c-u)^{2}}\right)+1\right)}, b=\sqrt{2\left(\left(\frac{16 t^{2}}{(c+u)^{2}}\right)+1\right)}$ and $c=\sqrt{16 t^{2}+u^{2}}$.

Table 2. Energy differences of the possible dimer transitions

\begin{tabular}{ll}
\hline Energy variation & Transition \\
\hline$u_{1}=\epsilon_{0}+t$ & $E_{1,2}=E_{1,3}=E_{4,6}=E_{4,8}=E_{5,7}=E_{5,8}=E_{9,14}=E_{9,15}$ \\
$u_{2}=\epsilon_{0}-t$ & $E_{1,4}=E_{1,5}=E_{2,6}=E_{2,8}=E_{3,7}=E_{3,8}=E_{9,12}=E_{9,13}$ \\
$u_{3}=\epsilon_{0}+t+u$ & $E_{4,9}=E_{5,9}=E_{6,14}=E_{7,15}=E_{8,14}=E_{8,15}=E_{12,16}=E_{13,16}$ \\
$u_{4}=\epsilon_{0}-t+u$ & $E_{2,9}=E_{3,9}=E_{6,12}=E_{7,13}=E_{8,12}=E_{8,13}=E_{14,16}=E_{15,16}$ \\
$u_{5}=\epsilon_{0}+t+\frac{u+c}{2}$ & $E_{4,10}=E_{5,10}$ \\
$u_{6}=\epsilon_{0}+t+\frac{u-c}{2}$ & $E_{4,11}=E_{5,11}$ \\
$u_{7}=\epsilon_{0}-t+\frac{u+c}{2}$ & $E_{2,10}=E_{3,10}$ \\
$u_{8}=\epsilon_{0}-t+\frac{u-c}{2}$ & $E_{2,11}=E_{3,11}$ \\
$u_{9}=\epsilon_{0}+t+u-\frac{u+c}{2}$ & $E_{10,14}=E_{10,15}$ \\
$u_{10}=\epsilon_{0}+t+u-\frac{u-c}{2}$ & $E_{11,14}=E_{11,15}$ \\
$u_{11}=\epsilon_{0}-t+u-\frac{u+c}{2}$ & $E_{10,12}=E_{10,13}$ \\
$u_{12}=\epsilon_{0}-t+u-\frac{u-c}{2}$ & $E_{11,12}=E_{11,13}$ \\
\hline
\end{tabular}

$E_{i, j}$ represents the transition from the state $i$ to the state $j$.

18th Brazilian Workshop on Semiconductor Physics (BWSP 2017)
In order to obtain the atomic Green's functions, the Zubarev equation [4] is used:

$$
g^{a t}(\omega)=e^{\beta \Omega} \sum_{i=1}^{12} \frac{m_{i}}{\omega-u_{i}}
$$

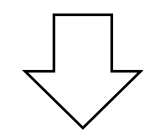

$$
g_{\sigma}^{a t}(\omega)=\left(\begin{array}{cccc}
g_{11} & g_{13} & 0 & 0 \\
g_{31} & g_{33} & 0 & 0 \\
0 & 0 & g_{22} & g_{24} \\
0 & 0 & g_{42} & g_{44}
\end{array}\right)
$$

Here, $m_{i}$ are the Green's functions residues and $u_{i}$ are the energy differences of the possible transitions. In the matricial form, the Green's functions are separated into two blocks: the upper left block is related to transitions that destroy a spin up electron and the lower right block is related to transitions that destroy a spin down electron.

The exact relation between the one particle cumulant and the one particle Green's function is:

$$
G(\boldsymbol{k} \sigma, i \omega)=\frac{M_{1 \sigma}(i \omega)}{1-\epsilon_{\boldsymbol{k}} M_{1 \sigma}(i \omega)}
$$

This relation is general and can be applied to the dimer. Then:

$$
\begin{aligned}
& g_{\sigma}^{\text {at }}(i \omega)=\frac{m_{1 \sigma}^{\text {at }}(i \omega)}{1-t m_{1 \sigma}^{\text {at }}(i \omega)} \\
& m_{1 \sigma}^{\text {at }}(i \omega)=\frac{g_{\sigma}^{\text {at }}(i \omega)}{1+t g_{\sigma}^{\text {at }}(i \omega)}
\end{aligned}
$$

Using a conduction band with a square shape, the Green's function for the lattice becomes:

$$
G(\omega)=\frac{1}{2 D} \int_{-D}^{D} d \epsilon \frac{M_{1 \sigma}(\omega)}{1-\epsilon M_{1 \sigma}(\omega)}=\frac{1}{2 D} \ln \left[\frac{1+D M_{1 \sigma}(\omega)}{1-D M_{1 \sigma}(\omega)}\right]
$$

This relation is exact, but the exact cumulants are unknown. The main approximation for the model is made here: the first order cumulant and the dimer solution are used as an approximation for the exact cumulants. Then, we obtain

$$
G(\omega)=\frac{1}{2 D} \ln \left[\frac{1+D m_{1 \sigma}^{\text {at }}(\omega)}{1-D m_{1 \sigma}^{\text {at }}(\omega)}\right]
$$


From the Green's functions for the lattice, the density of states [DOS] is obtained. A comparison between the DOS in the Hubbard I approximation and in our cumulant approach can be seen in the figure 2 :

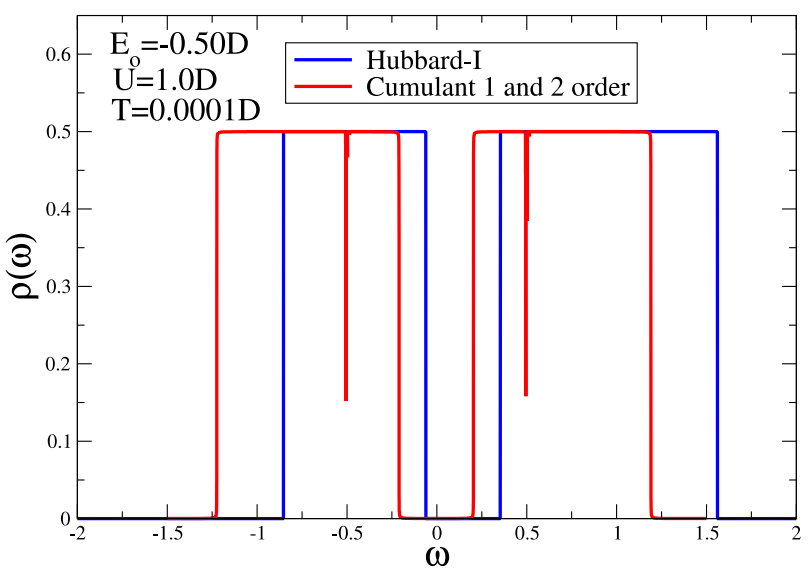

Figure 2. The density of states as a function of the electronic correlation $U$ for the Hubbard I approximation (blue) and our cumulant approach (red) taking into consideration both the single site cumulant and the dimer cumulant

We can also obtain the occupation numbers of the model through the relations:

$$
\begin{aligned}
n= & \left(\frac{1}{\pi}\right) \int_{-\infty}^{\infty} d z n_{F}(z) \operatorname{Im}\left(G^{\sigma}(z)\right), \\
n_{d}= & \left(\frac{1}{\pi}\right) \int_{-\infty}^{\infty} d z n_{F}(z) \operatorname{Im}\left(\Gamma^{\sigma}(z)\right) ; \\
& \left\langle\left\langle n_{i \bar{\sigma}} c_{i \sigma} ; c_{j \sigma}^{\dagger}\right\rangle\right\rangle_{\omega} \equiv \Gamma_{i j}^{\sigma}(\omega),
\end{aligned}
$$$$
n_{v a c}=\left(\frac{1}{\pi}\right) \int_{-\infty}^{\infty} d z\left(1-n_{F}\right) \operatorname{Im}\left(G^{\sigma}(z)\right)
$$

$$
n_{s}=n-n_{d}
$$

Such that

$$
C=n_{v a c}+n_{s}+n_{d}=1 .
$$

Where $n, n_{d}, n_{\text {vac }}, n_{s}$ and $C$ are, respectively, the single plus double occupation, the double occupation, the vacuum occupation, the single occupation and the completeness.

A comparison between occupation numbers of the model in different approaches can be seen in figures 3 and 4 :

18th Brazilian Workshop on Semiconductor Physics (BWSP 2017)

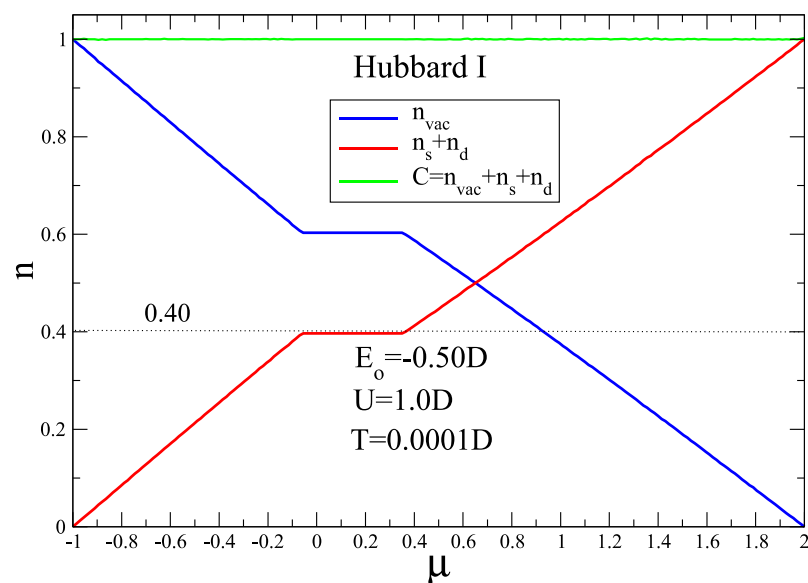

Figure 3. The occupation numbers of the model in the Hubbard I approximation

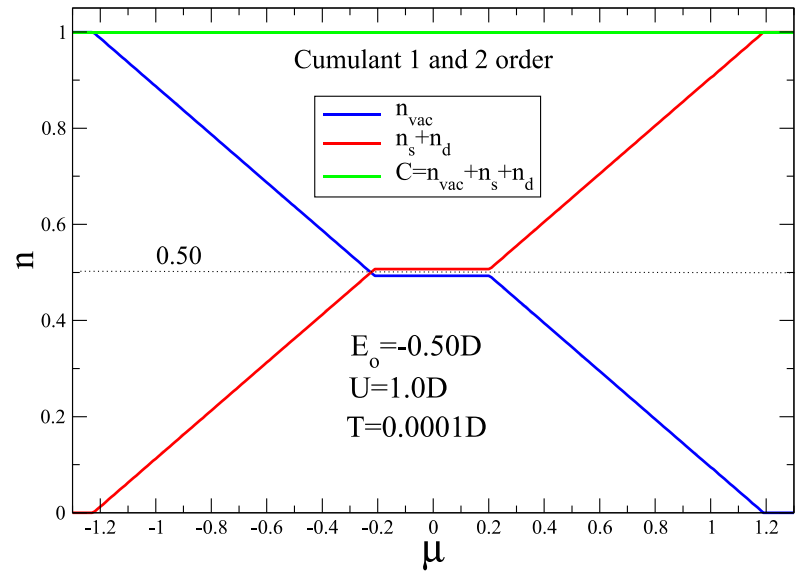

Figure 4. The occupation numbers of the model in the cumulant approach taking into consideration both the single site cumulant and the dimer cumulant

\section{Discussion}

The atomic method was implemented successfully to the Hubbard model, using the exact one site and two site solutions as a 'seed' for a cumulant expansion. The DOS and the occupation numbers for the model were obtained.

As a result of using both the first order cumulant and the dimer cumulant, the particle-hole symmetry for high values of electronic correlation was recovered, as can be seen by the analysis of the band areas on the DOS and by the analysis of the occupation numbers of the model for different values of the chemical potential.

In all the calculations we consider a square band of semi-width $D=1$ in a particle-hole symmetry case. $E_{o}=-0.5 \mathrm{D}$ is the local energy, $U=1.0 \mathrm{D}$ is the electron correlation and the temperature is $T=0.0001 \mathrm{D}$. 
In figure 2 we represent the DOS

in a typical particle-hole symmetric case, and we observe that the Hubbard I approximation does not fulfill this symmetry whereas our cumulant approach practically recovers this symmetry.

In figures 3 and 4 we plot the occupation numbers as functions of the chemical potential. The loss of the particle-hole symmetry in the Hubbard I approximation is reflected in the occupation number of the lower Hubbard band, which is equal to 0.4 as indicated in figure 3 . On the other hand, in figure 4 we obtain the occupation number 0.5 , which is consistent with the particle-hole symmetry of the present cumulant approach.

At the moment, we are developing a computational iterative algorithm to solve the Hubbard Hamiltonian for a cluster of $1,2,3, \ldots, N$ sites using this cumulant Green's function method and combine the solutions in order to improve the results. Also, by developing this algorithm, we would like to establish an alternative to the dynamical mean-field theory [DMFT] to solve the Hubbard Hamiltonian.

\section{Acknowledgments}

We would like to acknowledge CAPES and CNPq for the financial support.

\section{References:}

[1] J. Hubbard. Electron Correlations in Narrow Energy Bands. Proc. R. Soc. Lond. A 1963 256, doi: 10.1098/rspa.1963.0204, published 26 November 1963.

[2] T. Lobo, M. S. Figueira and M. E. Foglio, Nanotechnology. 21, 274007 (2010).

[3] Antoine Georges, Gabriel Kotliar, Werner Krauth, and Marcelo J. Rozenberg. Dynamical mean-field theory of strongly correlated fermion systems and the limit of infinite dimensions. Rev. Mod. Phys. 68, 13 Published 1 January 1996. doi: 10.1103/RevModPhys.68.13.

[4] Zubarev D N 1960 Sov. Phys.-Usp. 3320.

18th Brazilian Workshop on Semiconductor Physics (BWSP 2017) 